\title{
Les Phlébotomes (Diptera, Psychodidae) de la vallée du fleuve Sénégal.
}

\section{Première mention de Sergentomyia (Parvidens) lesleyae Lewis et Kirk, 1946 en Afrique de l'Ouest}

\author{
par J. P. DEDET, R. WINSHALL, R. D. HAYES ET P. DESJEUX \\ (Collaboration technique: A.-M. Simon et K. Mariko) \\ Institut Pasteur, B.P. 220, Dakar, Sénégal
}

et Public Health team, Garnett Fleming/O.M.V.S. environmental project, O.M.V.S. Dakar, Sénégal

\section{Résumée.}

Les auteurs présentent une collection de 2277 phlébotomes récoltés dans 26 stations régulièrement échelonnées le long du Fleuve Sénégal et réparties dans trois pays d'Afrique de l'Ouest : Mali, Mauritanie et Sénégal. Ils signalent la présence de Phlebotomus (Phlebotomus) duboscqi, vecteur de la leishmaniose cutanée, à l'orifice des terriers de rongeurs et mentionnent pour la première fois en Afrique de l'Ouest 3 exemplaires de Sergentomyia (Parvidens) lesleyae, phlébotome décrit du Soudan et uniquement signalé jusqu'à présent dans ce pays.

\section{Summary.}

The Phlebotomine sandflies (Diptera Psychodidae) frome the Senegal river valley. First record of Sergentomyia (Pardivens) lesleyae from West Africa.

The authors have collected 2277 phlebotomine sandflies from 26 sites located along the Senegal river, in three West African countries : Mali, Mauritania and Senegal. They noted the presence of Phlebotomus (Phlebotomus) duboscqi the cutaneous leishmaniasis vector in the region, at the opening of rodent burrows. Sergentomyia (Parvidens) lesleyae, a sandfly so far found only in Sudan, is now mentionned in West Africa for the first time.

\section{Introduction}

La connaissance des phlébotomes de l'Afrique de l'Ouest demeure encore à présent parcellaire. Un article synthétique d'Abonnenc et Larivière (1959) a rassemblé les captures ponctuelles rapportées çà et là dans la littérature par différents auteurs. Mais dans cette vaste région d'Afrique, aucun travail approfondi n'a porté sur les phlébotomes des foyers de leishmanioses, fort peu étudiés en eux-mêmes d'ailleurs.

Accepté le 28 juin 1979. 
En 1978, au cours de plusieurs missions épidémiologiques menées dans la vallée du Sénégal, nous avons pratiqué des récoltes régulières de phlébotomes dans 26 stations échelonnées le long du Fleuve. Il nous a paru utile de présenter cette collection provenant ensemble géngraphique homogène et fort neu étudié d'un point de vue entomologique. D'autant que la présence dans ce lot de phlébotomes de trois exemplaires (2 ô et 1 o) de Sergentomyia lesleyae correspond à la première mention de cette espèce en Afrique de l'Ouest.

\section{Matériel et Méthodes}

\section{I. - Région étudiée.}

Le fleuve Sénégal est constitué par la réunion à Bafoulabé (Mali) d'un bras important, le Bafing, naissant dans le Fouta-Djallon en Guinée et d'un affluent plus réduit, le Bakoye. Son cours s'étend sur 1800 kilomètres, entre le $10^{\mathrm{e}}$ et le $17^{\mathrm{e}}$ degrés de latitude nord. Fleuve tropical, son débit est important en saison des pluies (juin à octobre) et réduit pendant la saison sèche.

Les stations prospectées sont régulièrement échelonnées le long de la vallée du Sénégal, dans la portion malienne (11 stations) et sur les rives sénégalaise ( 6 stations) et mauritanienne ( 9 stations) du fleuve. Les 8 premières stations, les plus méridionales, sont situées dans la zone climatique tropicale à longue saison sèche, les autres dans la zone climatique sahélienne (fig. 1).

\section{II. - Technique de piégeage et biotopes inventoriés :}

Les captures ont été réalisées à l'aide de feuilles de papier enduites d'huile de ricin et déposées à l'ouverture des gîtes de repos des phlébotomes adultes.

Les principaux biotopes prospectés ont été les suivants : terriers de rongeurs, creux d'arbres, termitières et fissures d'érosion du sol. Les captures se sont échelonnées entre janvier et mai 1978 .

\section{III. - Montage et détermination :}

Les exemplaires récoltés ont été conservés dans l'alcool à $70^{\circ}$. Ils ont été éclaircis dans la soude à $20 \%$ pendant 3 heures, puis dans le Marc-André. Le montage a eu lieu dans le liquide de Hoyer. La détermination des femelles et de certains mâles a rendu nécessaire la dissection du pharynx et du cibarium de la majeure partie des exemplaires.

\section{Résultats et discussion}

Au cours de la présente prospection, 2277 exemplaires de phlébotomes ont été récoltés, se répartissant comme suit, en 13 espèces et une variété :

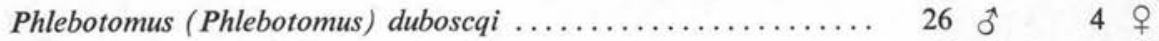
Phlebotomus (Anaphlebotomus) rodhaini................ 2 . 0 क 


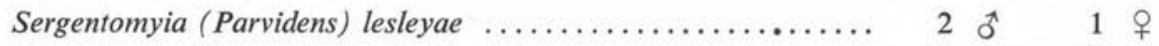

Sergentomyia (Sergentomyia) schwetzi.................. 65 o $\sigma^{1} \quad 90$ 우

Sergentomyia (Sergentomyia) schwetzi var. atypicus ......... $40 \quad \begin{gathered}\pi \\ 0\end{gathered}$

Sergentomyia (Sergentomyia) dubia .................. 396 o 618 우

Sergentomyia (Sergentomyia) antennata ................... 37 के 128 우

Sergentomyia (Sergentomyia) bedfordi .................. 25 ô. 40 우

Sergentomyia (Sergentomyia) buxtoni ................. 4 б $\sigma^{4} 4$ 우

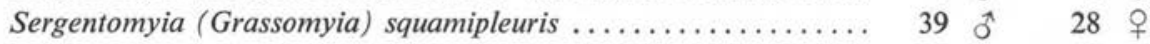

Sergentomyia (Parrotomyia) magna ..................... 108 ô 274 우

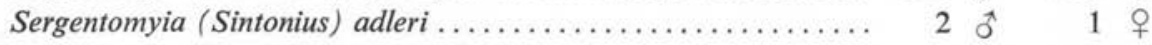

Sergentomyia (Sintonius) christophersi ................. . 1 우

Sergentomyia (Sintonius) clydei .................... 185 o

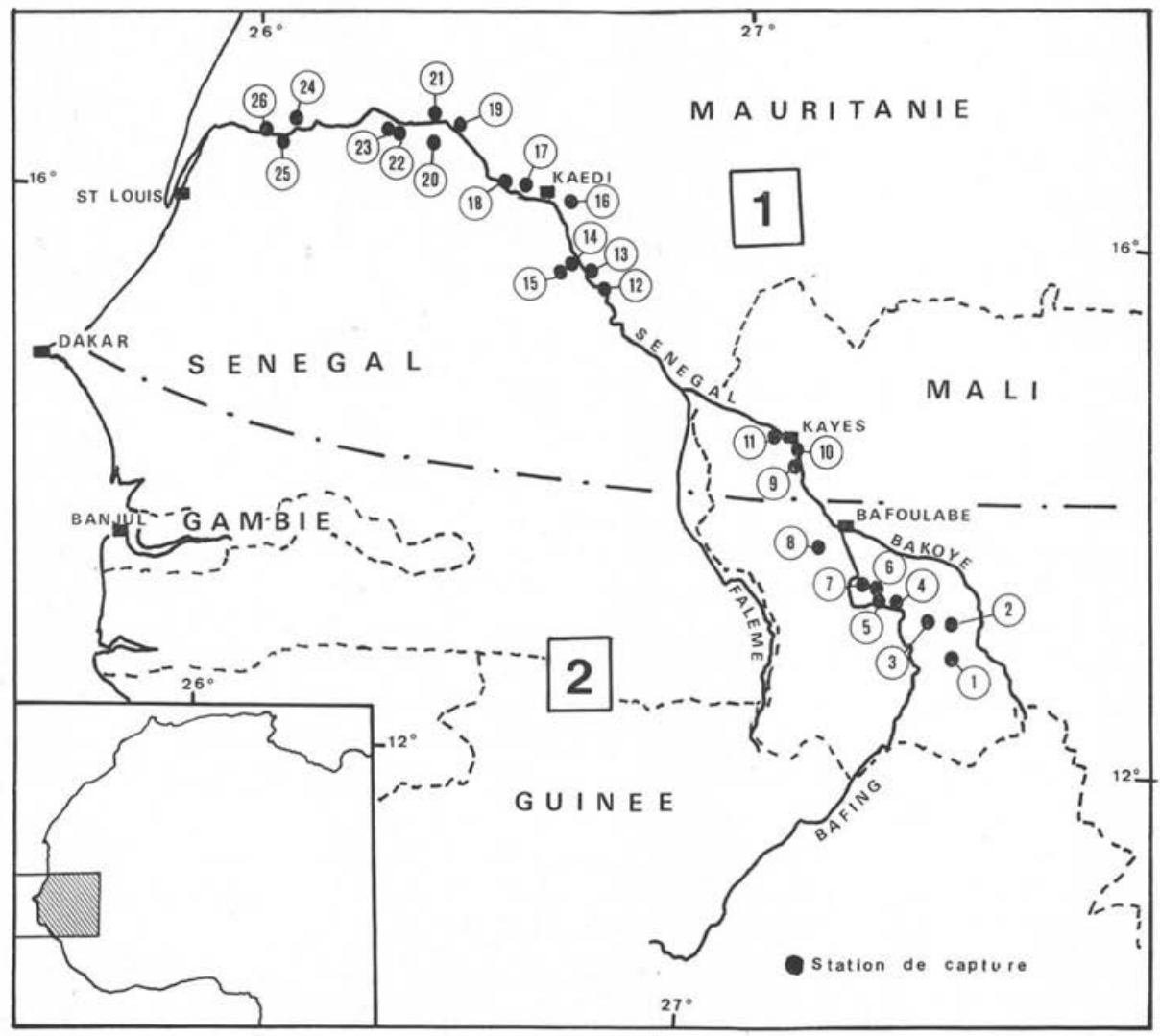

Fig. 1. Mise en place des stations prospectées dans la vallée du fleuve Sénégal. La ligne en traits-points sépare la zone climatique sahélienne (1) de la zone tropicale à longue saison sèche (2). 
D'une manière générale, notons que les espèces du genre Sergentomyia dominent tant par le nombre d'individus capturés que par la diversité (onze espèces différentes et une variété, se recrutant dans cinq sous-genres distincts).

La ventilation des captures en fonction des types de biotopes piégés apparaît sur le tableau I.

Tableau I. Répartition des espèces récoltées en fonction des types de biotopes pièges

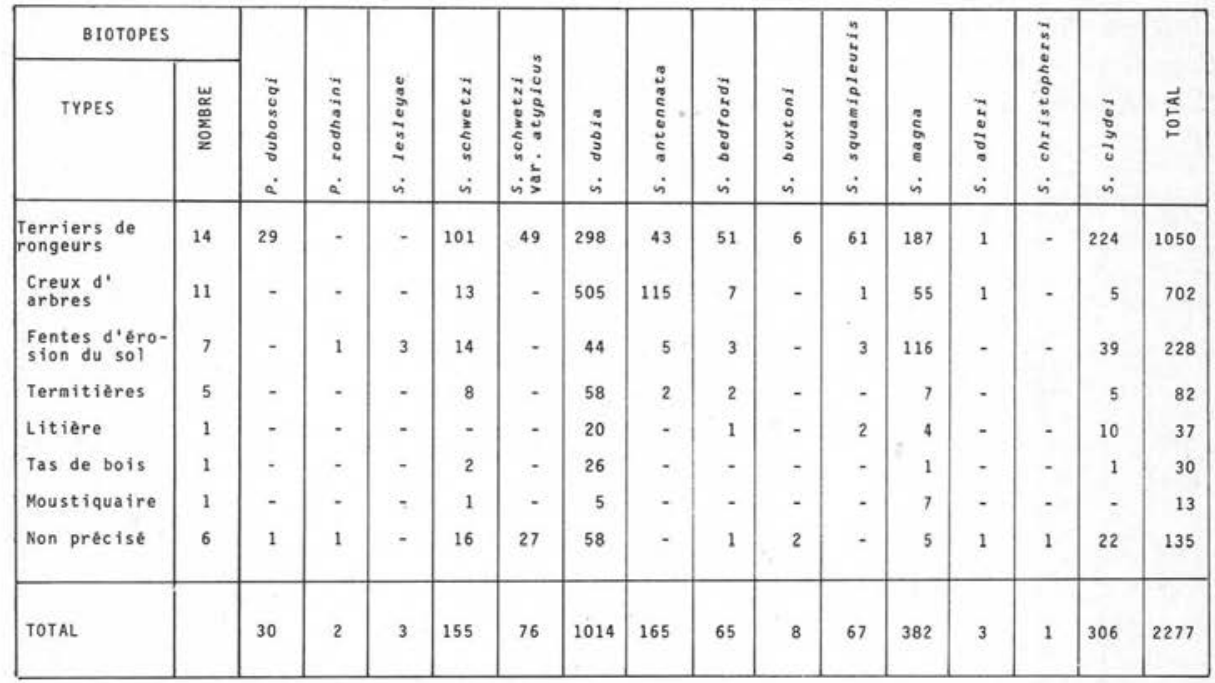

Terriers de rongeurs et creux d'arbres ont fourni les récoltes les plus abondantes et les plus variées. Douze espèces ont été récoltées à l'orifice des terriers de rongeurs, parmi lesquelles dominent numériquement $S$. dubia et $S$. clydei; $S$. magna et $S$. schwetzi y sont également nombreux. Phlebotomus duboscqi bien qu'assez faiblement représenté dans les captures, a été exclusivement récolté à l'orifice des terriers de rongeurs.

Dans les creux d'arbres, sur les huit espèces rencontrées, c'est le complexe $S$. dubia$S$. antennata qui domine. Les fentes d'érosion du sol se sont révélées de bons gîtes à phlébotomes ( 9 espèces, parmi lesquelles se signale $S$. magna).

En revanche, les termitières de cette région ont été trouvées assez peu peuplées si l'on compare ces résultats à ceux obtenus par Raymond et Cornet (1975) au Sénégal oriental et par Dedet et coll. (1978) dans la région de Thiès.

Le nombre précis de papiers huilés posés dans chaque station n'ayant pas été régulièrement noté, la disparité des résultats obtenus dans les deux étages bioclimatiques (272 exemplaires récoltés dans 8 stations de l'étage tropical à longue saison sèche pour 2005 exemplaires dans 18 stations de l'étage sahélien) ne saurait donner lieu à une quelconque interprétation écologique. 
Concernant les particularités morphologiques ou biologiques, nous ne retiendrons que les espèces méritant une attention spéciale.

\section{Phlebotomus (Phlebotomus) duboscqi.}

$P$. duboscqi fait preuve en Afrique de l'Ouest d'une affinité élective pour les terriers de rongeurs. Dedet et coll. (1978) ont insisté sur la présence de ce phlébotome dans ce biotope dans la région de Thiès (Sénégal), en pleine zone endémique de leishmaniose cutanée, et ont trouvé des femelles spontanément infectées par des Leishmania dont la caractérisation et le typage ont montré l'identité avec les souches isolées dans la même région à partir de rongeurs et de lésions humaines d'une part et avec Leishmania major d'autre part (Dedet et coll., 1979).

La présence de cette espèce, dans son biotope d'élection, aux confins sénégalo-mauritaniens constitue une intéressante donnée épidémiologique.

\section{Sergentomyil (Sintonius) clydei.}

L'abondance de $S$. clydei à l'orifice des terriers de rongeurs, déjà constatée dans la région de Thiès (Dedet et coll., 1978), attire l'attention sur cette espèce au comportement trophique polyvalent et dont l'anthropophilie a été notée à diverses reprises (Abonnenc, 1972).

\section{Sergentomyia (Parvidens) lesleyae.}

Phlébotome décrit par Lewis et Kirk en 1946 d'après un mâle et une femelle capturés à Kortala, dans la région des Monts Nuba au Soudan, il représente l'espèce type du sousgenre Parvidens qui comprend seulement deux autres espèces : Sergentomyia heischi Kirk et Lewis 1950 et Sergentomyia iranica Lewis et Mesghali 1961.

Ce sous-genre, d'abord rangé dans le genre Phlebotomus puis ramené à un groupe autonome, a enfin été rattaché au genre Sergentomyia par Theodor et Mesghali, en 1964.

Les exemplaires ( 2 ôt et 1 क $)$ que nous rapportons à $S$. lesleyae ont été capturés le 16 mai 1978 à l'orifice de fentes d'érosion du sol, près de la localité de N'Gorel en Mauritanie $\left(16^{\circ} 38^{\prime} \mathrm{N}-14^{\circ} 24^{\prime} \mathrm{W}\right)$. Nous donnons ci-dessous les principaux caractères morphologiques et les principales mensurations de ces spécimens.

\section{Mâle :}

taille : $2,21 \mathrm{~mm}$,

antenne : AIII $=0,12-0,13 \mathrm{~mm}<$ IV + V

$\mathrm{AIII} / \mathrm{E}=1,2$; épines géniculées courtes (fig. 2); formule : $2 / \mathrm{III}-\mathrm{XII}$ (segments

absents au-delà),

palpes : P1 $=0,08 \mathrm{~mm}$; P2 $=0,1 \mathrm{~mm}$; P3, P4 et P5 absents

cibarium et pharynx postérieur inermes,

aile : longueur $=1,13 \mathrm{~mm}$; largeur $=0,24 \mathrm{~mm}$; indice alaire $=0,23$; delta $=-0,07 \mathrm{~mm}$; alpha $=0,06 \mathrm{~mm}$; bêta $=0,26 \mathrm{~mm} ; \mathrm{pi}=0,1 \mathrm{~mm}$,

genitalia (fig. 2) : coxite $=0,23 \mathrm{~mm}$; style $=0,10 \mathrm{~mm}$ avec 5 épines ( 4 terminales en deux groupes et une médiane très longue); paramère $=0,19 \mathrm{~mm}$, bilobé, avec plaque Annales de Parasitologie humaine et comparée (Paris), t. 55, $\mathrm{n}^{\circ} 1$. 
additionnelle au lobe inférieur; lobe latéral $=0,19 \mathrm{~mm}$; fourreau pénien $=0,23 \mathrm{~mm}$ épais, digitiforme, à extrémité légèrement dépigmentée; pompe génitale de grande taille, située au IVe segment (fig. 2); rapport $\mathrm{FG} / \mathrm{PG}=4,75$.

\section{Femelle :}

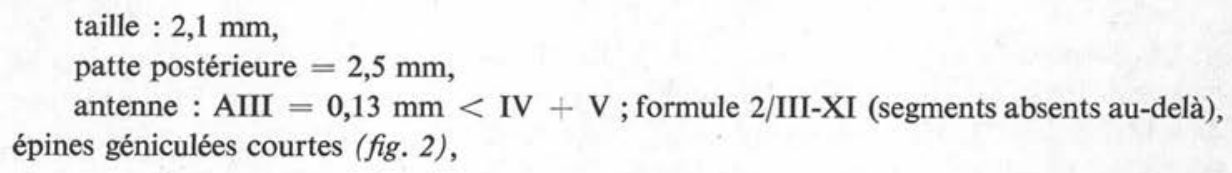
épines géniculées courtes (fig. 2),

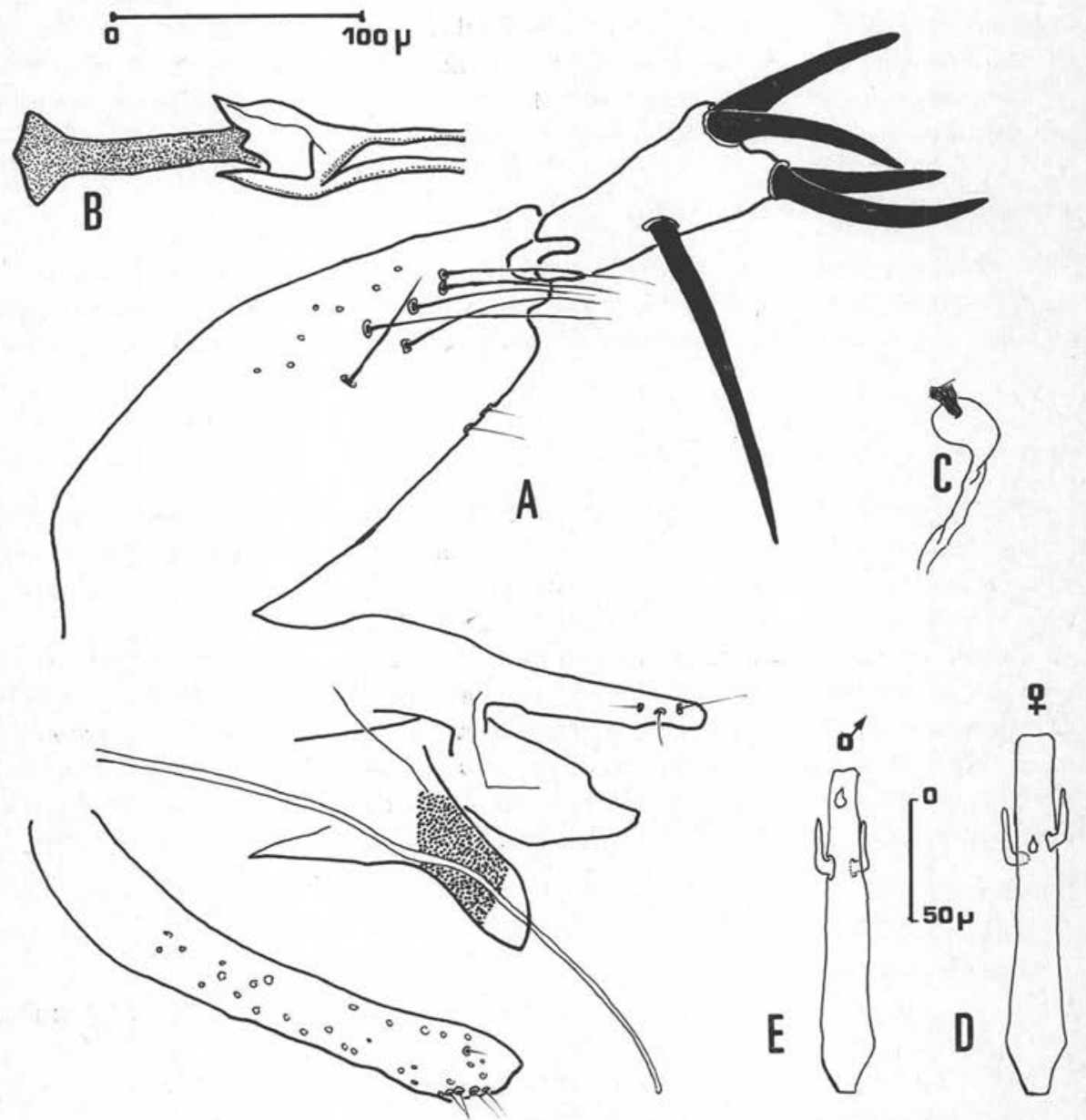

Fig. 2. Détails morphologiques des exemplaires de Sergentomyia lesleyae récoltés en Mauritanie. $A$ : appareil génital mâle;

$B$ : pompe génitale;

$C$ : spermathèque ; troisièmes segments des antennes mâles (E) et femelle (D). 
palpes : $\mathrm{P} 1=0,15 \mathrm{~mm} ; \mathrm{P} 2=0,18 \mathrm{~mm} ; \mathrm{P} 3=0,11 ; \mathrm{P} 4$ et $\mathrm{P} 5$ absents, pharynx postérieur armé d'une plage postérieure de dents fortes et acérées (fig. 3), aile : longueur $=2,07 \mathrm{~mm}$; largeur $=0,48 \mathrm{~mm}$; indice alaire $=0,3 ;$ delta $=-0,04 \mathrm{~mm}$; alpha $=0,09 \mathrm{~mm}$; bêta $=0,3 \mathrm{~mm} ; \mathrm{pi}=0,1 \mathrm{~mm}$, spermathèque : une seule visible; sacciforme et à parois lisses (fig. 2).

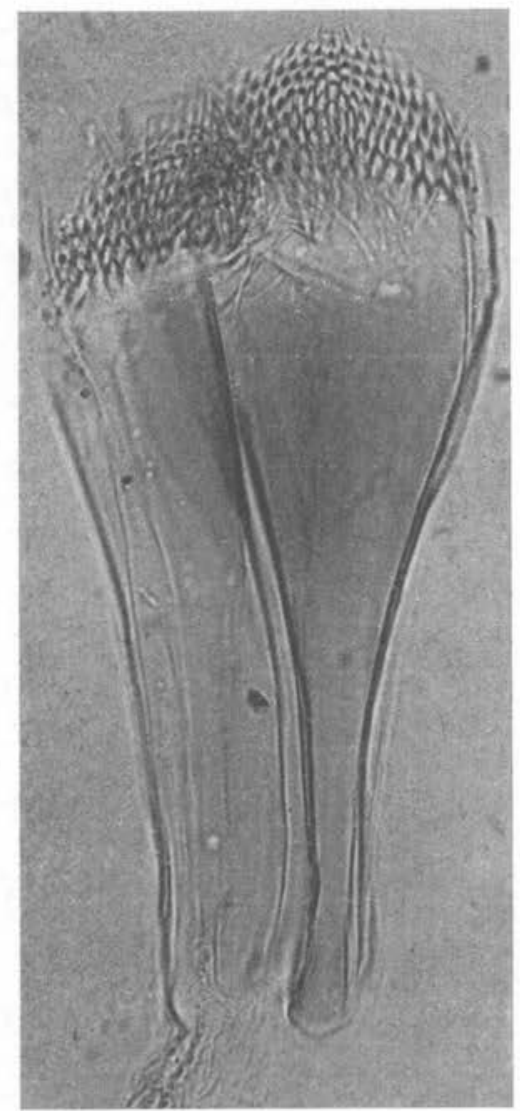

Fig. 3. Pharynx postérieur de l'exemplaire mauritanien de S. lesleyae.

La morphologie des génitalia et celle du pharynx sont absolument typiques et permettent de ranger les exemplaires mauritaniens dans le sous-genre Parvidens. La diagnose différentielle avec Sergentomyia heischi est également aisée : chez le mâle, le lobe inférieur des paramères est dépourvu des fortes épines que l'on rencontre chez $S$. heischi, mais au contraire porte une plaque additionnelle large et arrondie comme les exemplaires soudanais de $S$. lesleyae; chez la femelle, les épines géniculées sont courtes comme chez $S$. lesleyae et non longues et atteignant l'extrémité du segment antennaire, comme chez $S$. heischi. 
Seules des différences minimes ont été observées entre les exemplaires mauritaniens d'une part et les exemplaires soudanais décrits par Lewis et Kirk (1946). Elles portent essentiellement sur une superficie plus réduite de la plage de denticulations du pharynx femelle de l'exemplaire mauritanien (mais il s'agit vraisemblablement là d'une altération due à la dissection du pharynx de notre unique exemplaire). De même, certaines mensurations sont apparues légèrement supérieures chez les exemplaires mauritaniens (longueur totale, aile, troisième segment de l'antenne), mais elles sont à rapporter à des variations individuelles, régulièrement objervées au sein d'une même espèce, voire au sein de populations homogènes d'une même espèce. Au demeurant, nous identifions les trois exemplaires capturés dans le sud de la Mauritanie à $S$. lesleyae, dont il s'agit de la première mention hors du Soudan.

La répartition de cette espèce était jusqu'à présent limitée à plusieurs provinces de l'est de ce pays : Nil Bleu (Lewis et Kirk, 1957; Qutubuddin, 1961; Hoogstraal et Heyneman, 1969), Kassala (Qutubuddin, 1961), Kordofan et Haut Nil (Hoogstraal et Heyneman, 1961). Selon Qutubuddin, sa fréquence est élevée dans les zones biogéographiques de savane et de hautes terres abyssiniennes, correspondant d'ailleurs à des zones de kala-azar. S. lesleyae a été capturé au Soudan tout au long de l'année : janvier (Lewis et Kirk, 1946), avril, mai et août (Qutubuddin, 1961), octobre et novembre (Lewis et Kirk, 1957). En Mauritanie, nous l'avons récolté en fin de saison sèche, en région de savane sahélienne. Dans son biotope de capture (fentes d'érosion du sol), ce phlébotome était accompagné des espèces suivantes: $S$.

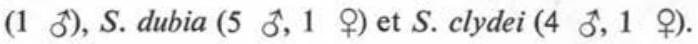

Trois autres types de biotopes piégés dans la même station (termitières, creux d'arbres et terriers de rongeurs) ne présentaient aucun autre exemplaire de S. lesleyae.

\section{Conclusion}

La découverte en Afrique de l'Ouest d'un phlébotome jusqu'alors réputé endémique du Soudan constitue une importante nouveauté et souligne, s'il en était besoin, la méconnaissance de la faune phlébotomienne de régions entières du continent africain.

\section{REMERCIEMENTS}

Nous remercions la Direction du British Museum (Histoire Naturelle) de Londres pour les facilités offertes, ainsi que le Docteur D. J. Lewis qui a bien voulu examiner nos exemplaires de $S$. lesleyae, a confirmé notre diagnose et a aimablement mis à notre disposition sa collection de $S$. lesleyae du Soudan.

\section{Bibliographie}

Abonnenc, E., 1972 : Les phlébotomes de la région éthiopienne (Diptera, Psychodidae). Mém. O.R.S.T.O.M., $55,290 \mathrm{p}$.

Abonnenc E., Larivière M., 1959 : Répartition des phlébotomes de l'Ouest africain (Diptera, Psychodidae), Bull. I.F.A.N., 21, 204-226. 
Dedet J. P., Derouin F., Cornet M., 1978 : Infestation spontanée de Phlebotomus duboscqi par des promastigotes de Leishmania au Sénégal. C.R. Acad. Sci., Paris, sér. D, 286, 301-302.

Dedet J. P., Derouin F., Hubert B., Chance M., Schnur L., 1979 : Isolation of Leishmania major from Mastomys erythroleucus and Tatera gambiana in Senegal (West Africa). Ann. Trop. Med. Parasit., 73, 433-437.

Hoogstraal H., Heyneman D., 1969 : Leishmaniasis in the Sudan Republic. 30. Final Epidemiologic Report. Am. J. Trop. Med. Hyg., 18, 1091-1210.

Kirk R., Lewis J. J., 1950 : Three new sandflies (Phlebotomus) from East Africa. Proc. R. Ent. Soc. Lond. (B.), 19, 11-13.

Lewis D. J., Kirk R., 1946: Five species of Phlebotomus (Diptera, Psychodidae) from the Sudan. Proc. R. Ent. Soc. Lond. (B.), 15, 55-60.

Lewis D. J., Kirk R., 1957 : Some Phlebotominae of the Sudan Republic. Ann. Mag. Nat. Hist., 10, 632-640.

Lewis D. J., Mesghali A., 1961 : A remarkable new phlebotomine sandfly (Diptera : Psychodidae) from Iran. Proc. R. Ent. Soc. Lond. (B.), 30, 121-123.

Qutubuddin M., 1961 : Notes on the Phlebotominæ of the Sudan Republic with the description of a new species and sub-species. Ann. Mag. Nat. Hist., 4, 593-611.

Raymond H. L., Cornet M, 1976 : Phlébotomes (Diptera, Psychodidae) des termitières du Sénégal OrientalAnn. Parasitol. Hum. Comp., 51, 259-262.

Theodor O., Mesghali A., 1964 : On the Phlebotominae of Iran. J. Med. Ent., 1, 285-300. 